\title{
On the initial conditions in continuous-time fractional linear systems
}

\author{
Manuel Duarte Ortigueira*,1 \\ UNINOVAIDEE, Faculdade de Ciências e Tecnologia da UNL, Campus da FCT da UNL, \\ Quinta da Torre, 2829-516 Caparica, Portugal
}

Received 1 December 2002

\begin{abstract}
The initial condition problem for fractional linear system initialisation is studied in this paper. It is based on the generalised initial value theorem. The new approach involves functions belonging to the space of Laplace transformable distributions verifying the Watson-Doetsch lemma. The fractional derivatives of these functions are independent of the derivative definition. This class includes the most important functions appearing in computing the Impulse Response of continuous-time fractional linear systems.
\end{abstract}

(C) 2003 Elsevier B.V. All rights reserved.

Keywords: Initial value problem; Fractional differintegration

\section{Introduction}

The increase in the number of physical and engineering processes that are found to be best described by fractional differential equations has been motivation for the study and application of fractional calculus. The effective application of the fractional calculus to science and engineering problems needs a coherent fractional systems theory. In previous papers $[11,12]$ we tried to do some contribution to that goal. However, a problem that seemed to be already solved originated an interesting discussion [1,5-7,13]: the initialisation

\footnotetext{
* Tel.: +21-294-8520; fax: +21-295-7786.

E-mail address: mdo@uninova.pt (M.D. Ortigueira).

${ }^{1}$ Also with INESC, R. Alves Redol, 9, 1000-029 Lisboa, Portugal.
}

problem. The reason is in two facts:

(a) Two different solutions are known.

(b) They both seem to be unsatisfactory.

Lorenzo and Hartley showed that the proper initialisations of fractional differintegrals are non-constant functions, generalising the integer order case. They have treated the issue of initialisation in several papers where they formulated the problem correctly, analysed the effect of a wrong initialisation and proposed solutions [5-7].

In this paper, we will approach the problem from a different point of view having in mind:

(a) the class of distributions having Laplace Transform (LT),

(b) the initial value theorem,

(c) the Watson-Doetsch lemma and 
(d) the way how the initial values appear in the differentiation process.

The paper proceeds as follows. The initial value problem is treated in several steps (Section 2) by

(a) enunciating the initial value theorem;

(b) doing a simple reasoning;

(c) presenting the initial value theorem.

In Section 3, we present our approach to the solution, beginning by the Watson-Doetsch lemma [3] that allows us to introduce a class of functions where we will look for our solutions. These are obtained through a step by step differentiation. Within this framework, we show that the common approaches based on the Riemann-Liouville or Caputo definitions are particular cases. At last, we exemplify and present some conclusions.

\section{On the initialisation problem}

\subsection{Statement of the problem}

Let us assume that we have a fractional linear system described by a fractional differential equation like:

$$
\begin{aligned}
& \sum_{n=0}^{N} a_{n} D^{v_{n}} y(t)=\sum_{m=0}^{M} b_{m} D^{v_{m}} x(t), \\
& v_{n}<v_{n+1},
\end{aligned}
$$

where $D$ means derivative and $v_{n} n=0,1,2, \ldots$ are derivative orders that we will assume to be positive real numbers. The initialisation problem appears when we try to compute the output of the system to a given input applied at a time instant: we must specify the initial state of the system.

In problems with non-zero initial conditions it is a common practice to introduce the one-sided Laplace transform. However, there is no particular justification for such introduction. The initial conditions must appear independently of using or not a transform. In fact, we intend to solve a given differential equation (1) for values of $t$ greater than a given initial instant, that, without loosing generality, we can assume to be the origin. To treat the question, it is enough to multiply both members of the equation by the unit step Heaviside function, $u(t)$, and rearrange the equation terms as shown next with a simpler example. Consider the ordinary constant coefficient differential equation:

$y^{(N)}(t)+a y(t)=x(t) \quad N \in Z_{0}^{+}$.

Assume that the products $y^{(N)}(t) u(t)$ and $x(t) u(t)$ can be considered as distributions [2] and that we want to solve Eq. (2) for $t>0$. The multiplication by $u(t)$ leads to

$y^{(N)}(t) u(t)+a y(t) u(t)=x(t) u(t)$.

Thus, we have to relate $y^{(N)}(t) u(t)$ with $[y(t) u(t)]^{(N)}$. This can be done recursively provided that we account for the properties of the distribution $\delta(t)$ and its derivatives. We obtain the well known result:

$$
\begin{aligned}
y^{(N)}(t) u(t)= & {[y(t) u(t)]^{(N)} } \\
& -\sum_{i=0}^{N} y^{(N-1-i)}(0) \delta^{(i)}(t)
\end{aligned}
$$

that states that $y^{(N)}(t) u(t)=[y(t) u(t)]^{(N)}$ for $t>0$. They are different at $t=0$. This is the reason why we speak in initial values as being equivalent to initial conditions. In the above equation we have

$$
\begin{aligned}
& {[y(t) u(t)]^{(N)}+a[y(t) u(t)]} \\
& \quad=x(t)+\sum_{i=0}^{N-1} y^{(N-1-i)}(0) \delta^{(i)}(t) .
\end{aligned}
$$

The initial conditions appear naturally, without using any transform. It is interesting to remark that the second term on the right in (4) is constituted by the derivatives of the Heaviside functions that we are needing for making continuous the left hand function before computing the derivative. For example, $y(t) u(t)$ is not continuous at $t=0$, but $y(t) u(t)-y(0) u(t)$ is; so, its derivative is given by $[y(t) u(t)]^{\prime}-y(0) \delta(t)$. The process is repeated.

In fractional case, the problem is similar, but it is not so clear the introduction of the initial conditions, because the involved functions can be infinite at $t=0$.

\subsection{Some facts}

When computing a $\alpha$ order derivative, it is well known, that [13]

(1) Different derivative definitions imply different steps in going form 0 to $\alpha$ (see appendix). 
(2) Different steps lead to different initial values.

(3) In the differentiation steps some orders and corresponding initial values are fixed and defined by the equation: in the left-hand side in (1) when "going" from 0 to $v_{N}$, we have to "pass" by all the $v_{i}(i=$ $1, \ldots, N-1)$-with the corresponding initial conditions. However we can compute other derivatives of orders $\gamma_{i}\left(v_{i}<\gamma_{i}<v_{i+1}\right)$ that introduce initial conditions too.

(4) If in (1) all the $v_{n}$ are rational numbers, the differential equation can always be written as $[9,11]$

$\sum_{n=0}^{N} a_{n} D^{n v} y(t)=\sum_{m=0}^{M} b_{m} D^{m v} x(t)$

leading as to conclude that the "natural" initial values will be $\left.D^{n v} y(t)\right|_{t=0+}$ for $n=0, \ldots, N-1$ and $\left.D^{n v} x(t)\right|_{t=0+}$ for $n=0, \ldots, M-1$.

(5) Independently of the way followed to compute a given derivative, the Laplace Transform of the derivative satisfy: $\operatorname{LT}\left[D^{\alpha} f(t)\right]=s^{\alpha} \operatorname{LT}[f(t)]$. So, the different steps in the derivative computation correspond to different decompositions of the number $\alpha$ :

$\alpha=\sum_{i} \sigma_{i}$.

These considerations lead us to conclude that the initial condition problem in the fractional case has infinite solutions.

\subsection{An example}

In practical applications we can find several examples of systems with Transfer Functions given by

$H(s)=\frac{Q}{s^{\alpha}}$,

where $Q$ is a constant and $-1<\alpha<1$. They are known as "constant phase elements" $[4,8]$. In particular, the supercapacitors [8] are very important. The capacitor case is well studied by Westerlund [15], where he shows that the "natural" initial value is the voltage at $t=0$ that influences the output of the system through an initial function proportional to $t^{-\alpha} u(t)$.

With this example we had in mind to remark that the structure of the problem may lead us to decide what initial condition we should use - it is an engineering problem, not mathematical.

\subsection{The initial-value theorem}

The Abelian initial value theorem [16] is a very important result in dealing with the Laplace Transform. This theorem relates the asymptotic behaviour of a causal signal, $\varphi(t)$, as $t \rightarrow 0+$ to the asymptotic behaviour of $\Phi(\sigma)=\mathrm{LT}[\varphi(t)]$, as $\sigma=\operatorname{Re}(s) \rightarrow+\infty$.

The initial-value theorem: Assume that $\varphi(t)$ is a causal signal such that in some neighbourhood of the origin is a regular distribution corresponding to an integrable function. Also, assume that there is a real number $\beta>-1$ such that $\lim _{t \rightarrow 0+} \varphi(t) / t^{\beta}$ exists and is a finite complex value. Then

$\lim _{t \rightarrow 0+} \frac{\varphi(t)}{t^{\beta}}=\lim _{\sigma \rightarrow \infty} \frac{\sigma^{\beta+1} \Phi(\sigma)}{\Gamma(\beta+1)}$.

For proof see [16].

Let $-1<\alpha<\beta$. Then

$\lim _{t \rightarrow 0+} \frac{\varphi(t)}{t^{\alpha}}=\lim _{t \rightarrow 0+} \frac{\varphi(t)}{t^{\beta}} \frac{t^{\beta}}{t^{\alpha}}=0$

because the first factor has a finite limit given in (8) and the second zero as limit. Similarly, if $\beta<\alpha$,

$\lim _{t \rightarrow 0+} \frac{\varphi(t)}{t^{\alpha}}=\infty$.

This suggests us that, near $t=0, \varphi(t)$ must have the format: $\varphi(t)=s(t) t^{\beta} u(t)$, where $s(t)$ is regular at $t=0$.

\section{The proposed solution}

\subsection{The Watson-Doetsch class}

Let us consider the class of functions with Laplace Transform analytic for $\operatorname{Re}(s)>\gamma$. To the subclass of functions such that

$\varphi(t) \approx t^{\beta} \sum_{n=0}^{\infty} a_{n} \frac{t^{n v} u(t)}{\Gamma(\beta+1+n v)}$

as $t \rightarrow 0+$ where $\beta>-1$ and $v>0$. The powers have their principal values. For our applications to differential equations, we will assume that $v$ is greater than the maximum derivative order. The Watson-Doetsch 
lemma [3], states that the LT $\Phi(s)$ of $\varphi(t)$ satisfies

$\Phi(s) \approx \frac{1}{s^{\beta+1}} \sum_{n=0}^{\infty} a_{n} \frac{1}{s^{n v}}$

as $s \rightarrow \infty$ and $\operatorname{Re}(s)>0$.

As it is clear, these functions verify the initial value theorem. On the other hand, $\varphi(t)$ in (11) has a format very common in solving the fractional differential equations. In fact, the impulse response of the equation

$D^{\alpha} y(t)+a y(t)=x(t)$

is given by

$h(t)=\sum_{n=1}^{\infty}(-a)^{n-1} \frac{t^{n \alpha-1} u(t)}{\Gamma(n \alpha)}$

as it can be verified. For this reason, we will use "=" instead of " $\approx$ " in (11) and (12). On the other hand, as $\sigma^{\beta} \Phi(\sigma)=\operatorname{LT}\left[D^{\beta} \varphi(t)\right]$,

$\lim _{\sigma \rightarrow \infty} \sigma\left[\sigma^{\beta} \Phi(\sigma)\right]=\left.D^{\beta} \varphi(t)\right|_{t=0+}$

by the usual initial value theorem. So,

$\left.D^{\beta} \varphi(t)\right|_{t=0+}=\lim _{\sigma \rightarrow \infty} \sigma^{\beta+1} \Phi(\sigma)$

that is a generalisation of the usual initial value theorem, obtained when $\beta=0$. Here, we introduce the impulse response of the differintegrator, $\delta^{(\alpha)}(t)=\mathrm{LT}^{-1}\left[s^{\alpha}\right]$, given by

$\delta^{(\alpha)}(t)= \begin{cases}\frac{t^{-\alpha-1}}{\Gamma(-\alpha)} u(t), & v \neq 0, \\ \delta^{(n)}(t), & v=0,\end{cases}$

where $\alpha=n-v$, with $0 \leqslant v<1$ \{see the appendix . Because $u(t)=D^{\beta}\left[t^{\beta} u(t)\right] / \Gamma(\beta+1)$ and using (8), we obtain

$\lim _{t \rightarrow 0+} \frac{\varphi(t)}{t^{\beta}}=\lim _{t \rightarrow 0^{+}} \frac{D^{\beta} \varphi(t)}{D^{\beta}\left[t^{\beta} u(t)\right]}=\frac{\varphi^{(\beta)}(0+)}{\Gamma(\beta+1)}$,

that is very similar to the usual l'Hôpital rule used to solve the $0 / 0$ problems.

Now, let us assume that $\varphi(t)$ is written as

$\varphi(t)=t^{\beta} f(t) u(t)$, where $f(t)$ is given by

$f(t)=\sum_{n=0}^{\infty} a_{n} \frac{t^{n v} u(t)}{\Gamma(\beta+1+n v)}$.

Attending to Eqs. (7)-(9), it is not hard to conclude that, when $t \rightarrow 0+$, we have

$\left.D^{\alpha} \varphi(t)\right|_{t=0+}= \begin{cases}0 & \text { if } \alpha<\beta, \\ f(0+) \Gamma(\beta+1) & \text { if } \alpha=\beta, \\ \infty & \text { if } \alpha>\beta .\end{cases}$

All the derivatives of order $\alpha<\beta$ have a zero initial value, while all the derivatives of order greater than $\beta$ are infinite at $t=0$. To obtain a continuous function we have to remove a term proportional to $t^{\beta-\alpha} u(t)$. This is important in dealing with differential equations and will be done in the following solution. We must remark that the previous results are valid independently of the fractional derivative definition we use. Eq. (21) shows also that the integration introduces zero initial condition.

Return back to Eq. (1). The previous considerations lead us to state for $y(t)$ - and similarly for $x(t)$ - the following format:

$y(t)=\sum_{k=0}^{N} f_{n}(t) t^{\gamma_{n}} u(t)$,

where $0<\gamma_{n}<\gamma_{n+1}$-according to the initial value theorem, we could use $-1<\gamma_{n}$, but in our present application it does not interest. $N$ is a positive integer that may be infinite, and the functions $f_{n}(t)(n=0, \ldots, N)$ and their derivatives of orders less than or equal to $\gamma_{N}$ are assumed to be regular at $t=0$. We may assume them to be given by (20).

\subsection{Step by step differentiation}

It is interesting to see how the initial values appear and their meaning. Let $y(t)$ be a signal given by (22). Let us introduce a sequence $\beta_{n}$ by

$\beta_{n}=\gamma_{n}-\sum_{k=0}^{n-1} \beta_{k}, \quad \beta_{0}=\gamma_{0}$.

Let us see what happens proceeding step by step.

(a) According to our assumptions $\beta_{0}$ is the least real for which $\lim _{t \rightarrow 0^{+}} y(t) / t^{\beta_{0}}$ is finite and nonzero. 
Let it be $y^{\left(\beta_{0}\right)}(0+) / \Gamma\left(\beta_{0}+1\right)$. All the derivatives $D^{\alpha} y(t)\left(\alpha<\beta_{0}\right)$ are continuous at $t=0$ and assume a zero value. The $\beta_{0}$ order derivative assumes the value $y^{\left(\beta_{0}\right)}(0+)$ and we can construct the function

$\varphi^{\left(\beta_{0}\right)}(t)=[y(t) u(t)]^{\left(\beta_{0}\right)}-y^{\left(\beta_{0}\right)}(0+) u(t)$,

that is continuous and assumes a zero value at $t=0$.

(b) Now, $\beta_{1}$ is the least real for which $\lim _{t \rightarrow 0^{+}} \varphi^{\left(\beta_{0}\right)}(t) / t^{\beta_{1}}$ is finite and non-zero. Let it be $y^{\left(\beta_{0}+\beta_{1}\right)}(0+) / \Gamma\left(\beta_{1}+1\right)$. Thus $\beta_{1}$ derivative of $\varphi^{\left(\beta_{0}\right)}(t)$ is given by

$$
\begin{aligned}
\varphi^{\left(\beta_{0}+\beta_{1}\right)}(t)= & {[y(t) u(t)]^{\left(\beta_{0}+\beta_{1}\right)} } \\
& -y^{\left(\beta_{0}\right)}(0+) \delta^{\left(\beta_{1}-1\right)}(t) \\
& -y^{\left(\beta_{0}+\beta_{1}\right)}(0+) u(t)
\end{aligned}
$$

is again continuous at $t=0$.

(c) Again $\beta_{2}$ is the least real for which $\lim _{t \rightarrow 0^{+}} \varphi^{\left(\beta_{0}+\beta_{1}\right)}(t) / t^{\beta_{2}}$ is finite and non-zero. Let it be $y^{\left(\beta_{0}+\beta_{1}+\beta_{2}\right)}(0+) / \Gamma\left(\beta_{2}+1\right)$. Thus

$$
\begin{aligned}
f^{\left(\beta_{0}+\beta_{1}+\beta_{2}\right)}(t)= & {[y(t) u(t)]^{\left(\beta_{0}+\beta_{1}+\beta_{2}\right)} } \\
& -y^{\left(\beta_{0}\right)}(0+) \delta^{\left(\beta_{1}+\beta_{2}-1\right)}(t) \\
& -y^{\left(\beta_{0}+\beta_{1}\right)}(0+) \delta^{\left(\beta_{2}-1\right)}(t) \\
& -y^{\left(\beta_{0}+\beta_{1}+\beta_{2}\right)}(0+) u(t)
\end{aligned}
$$

is again continuous at $t=0$.

(d) Continuing with this procedure, we obtain a function:

$$
\begin{aligned}
\varphi^{\left(\gamma_{N}\right)}(t)= & {[y(t) u(t)]^{\left(\gamma_{N}\right)} } \\
& -\sum_{0}^{N-1} y^{\left(\gamma_{m}\right)}(0+) \delta^{\left(\gamma_{N}-\gamma_{i}-1\right)}(t),
\end{aligned}
$$

that is not continuous at $t=0$, but it can be made continuous if we subtract it $y^{\left(\gamma_{N}\right)}(0+) u(t)$. Eq. (27) states the general formulation of the initial value problem solution. As we can see, the initial values prolong their action for every $t>0$. This means that we have a memory about the initial conditions that decreases very slowly. Using the LT, we obtain

$$
\operatorname{LT}\left[\varphi^{\left(\gamma_{N}\right)}(t)\right]=s^{\gamma_{N}} Y(s)-s^{\gamma_{N}} \sum_{0}^{N-1} y^{\left(\gamma_{m}\right)}(0+) s^{-\gamma_{i}-1},
$$

that is a generalization of the usual formula for introducing the initial condition.

\subsection{Coherence test}

To verify the coherence of the result, we are going to study some special cases:

(1) $\gamma_{i}=i$, for $i=0,1, \ldots, N$.

We have: $\beta_{0}=0, \beta_{i}=1$, for $i=1, \ldots, N$ and

$$
\begin{aligned}
\varphi^{(N)}(t)= & {[y(t) u(t)]^{(N)} } \\
& -\sum_{0}^{N-1} y^{(m)}(0+) \delta^{(N-m-1)}(t) .
\end{aligned}
$$

Applying the LT to both members we obtain

$$
\operatorname{LT}\left[\varphi^{(N)}(t)\right]=s^{N} Y(s)-\sum_{0}^{N-1} y^{(m)}(0+) s^{N-m-1},
$$

that is the usual formula for the initial value problem. It is clear that $\varphi^{(N)}(t)=[y(t) u(t)]^{(N)}$ for $t>0$.

(2) $\gamma_{i}=\gamma+i$, for $i=0,1, \ldots, N$.

We obtain: $\beta_{0}=\gamma, \beta_{i}=1$, for $i=1, \ldots, N-1$. Then,

$$
\begin{aligned}
\varphi^{(N+\gamma)}(t)= & {[y(t) u(t)]^{(N+\gamma)} } \\
& -\sum_{0}^{N-1} y^{(m+\gamma)}(0+) \delta^{(N-1-m)}(t)
\end{aligned}
$$

and

$$
\begin{aligned}
\operatorname{LT}\left[\varphi^{(N+\gamma)}(t)\right]= & s^{N+\gamma} Y(s) \\
& -\sum_{0}^{N-1} y^{(m+\gamma)}(0+) s^{N-m-1}
\end{aligned}
$$

with $\gamma=0$, we obtain (30) again. With $\alpha=N+\gamma$, Eq. (32) can be rewritten as

$\operatorname{LT}\left[\varphi^{(\alpha)}(t)\right]=s^{\alpha} Y(s)-\sum_{0}^{N-1} y^{(\alpha-1-i)}(0+) s^{i}$,

that is the Riemann-Liouville solution.

(3) Putting $\gamma_{i}=i, i=0, \ldots, N-1, \gamma_{N}=N-\varepsilon$, $0<\varepsilon<1$, and $\alpha=N-\varepsilon$, we obtain $\varphi^{(\alpha)}(t)=[y(t) u(t)]^{(\alpha)}-\sum_{0}^{N-1} y^{(m)}(0+) \delta^{(\alpha-m)}(t)$,

that is similar to the Caputo solution. We will return to this later. 
(4) $\gamma_{i}=i \gamma$, for $i=0,1, \ldots, N$.

We have: $\beta_{0}=0, \beta_{i}=\gamma$, for $i=1, \ldots, N-1$. Then,

$$
\begin{aligned}
\varphi^{(N \gamma)}(t)= & {[y(t) u(t)]^{(N \gamma)} } \\
& -\sum_{0}^{N-1} y^{(m \gamma)}(0+) \delta^{((N-1-m) \gamma)}(t)
\end{aligned}
$$

giving

$\operatorname{LT}\left[\varphi^{(N \gamma)}(t)\right]=s^{N \gamma} Y(s)-\sum_{0}^{N-1} y^{(m \gamma)}(0+) s^{(N-m) \gamma-1}$

different from the results obtained with the one-sided LT and both Riemann-Liouville or Caputo differintegrations. This case is suitable for easy solution of equations of type (6).

\subsection{The Caputo case}

The Caputo case is not in the framework considered in Section 3.2. In fact, we considered there that the $\gamma_{n}(n=0, \ldots, N)$ is an increasing sequence. In Caputo differentiation, we have $\gamma_{n}=n$ for $(n=0, \ldots, N)$ and $\gamma_{N+1}=N-\varepsilon$ with $0<\varepsilon<1$. So, it is a sequence of order one derivatives followed by a fractional integration. As the integration does not introduce non-zero initial conditions, we have:

$$
\begin{aligned}
\varphi^{\left(\gamma_{N}\right)}(t)= & {[y(t) u(t)]^{\left(\gamma_{N}\right)} } \\
& -\sum_{0}^{N} y^{(i)}(0+) \delta^{(N-i-1-\varepsilon)}(t)
\end{aligned}
$$

or, putting $\alpha=N-\varepsilon$;

$\varphi^{(\alpha)}(t)=[y(t) u(t)]^{(\alpha)}-\sum_{0}^{N} y^{(i)}(0+) \delta^{(\alpha-i-1)}(t)$.

This can be generalized by introducing other integrations.

\subsection{Examples}

Consider the system described by Eq. (13) with $\alpha=3 / 2$. As in the equation we only have two terms we are not constrained and can choose any "way" to go from 0 to $\alpha$. We are going to consider four cases:

(1) $\gamma_{i}=3 / 2 i(i=0,1)$ or $\beta_{0}=0$ and $\beta_{1}=3 / 2$. From (41), we have

$\operatorname{LT}\left[\varphi^{(3 / 2)}(t)\right]=s^{3 / 2} Y(s)-y(0+) s^{1 / 2}$.

The free term is then

$\Phi_{f}(s)=y(0+) \frac{s^{1 / 2}}{s^{3 / 2}+a}$.

(2) $\gamma_{i}=1 / 2 i(i=0,1,2,3)$ or $\beta_{0}=0$ and $\beta_{i}=1 / 2$ $(i=1,2,3)$. We have now:

$$
\begin{aligned}
\operatorname{LT}\left[\varphi^{(3 / 2)}(t)\right]= & s^{3 / 2} Y(s) \\
& -\sum_{0}^{2} y^{(m / 2)}(0+) s^{(3-m) / 2-1}
\end{aligned}
$$

with

$\Phi_{f}(s)=\frac{\sum_{0}^{2} y^{(m / 2)}(0+) s^{(3-m) / 2-1}}{s^{3 / 2}+a}$

as the corresponding free term.

(3) $\gamma_{i}=1 / 2+i(i=0,1)$ or $\beta_{0}=1 / 2$ and $\beta_{1}=3 / 2$, giving the Riemann-Liouville solution:

$\operatorname{LT}\left[\varphi^{(3 / 2)}(t)\right]=s^{3 / 2} Y(s)-y^{(1 / 2)}(0+)$.

The same solution can be obtained with $\gamma_{I}=-1 / 2+$ $i(i=0,1,2)$. Now, the free term is given by

$\Phi_{f}(s)=y^{(1 / 2)}(0+) \frac{1}{s^{3 / 2}+a}$.

(4) $\gamma_{i}=i(i=0,1)$ and $\gamma_{2}=2-1 / 2$. It comes

$\operatorname{LT}\left[\varphi^{(3 / 2)}(t)\right]=s^{3 / 2} Y(s)-\sum_{0}^{1} y^{(m)}(0+) s^{3 / 2-m-1}$

giving the free term

$\Phi_{f}(s)=\frac{\sum_{0}^{1} y^{(m)}(0+) s^{(3 / 2-m-1)}}{s^{3 / 2}+a}$.

The situation is somehow different if we have an intermediary term as it is the case of the equation:

$y^{(\alpha)}(t)+a y^{(1)}(t)+b y(t)=x(t)$.

Now, when going from $\gamma=0$ to $3 / 2$, we have to "pass" by $\gamma=1$. Obviously, we can force the corresponding initial value to be zero. 
It is interesting to see what happens when we consider an ordinary integer order differential equation as a special case of a fractional differential equation. Consider the simple case:

$y^{\prime}(t)+a y(t)=x(t)$.

Putting $\gamma_{i}=1 / 2 i(i=0,1,2)$, we have

$f^{\prime}(t)=[y(t) u(t)]^{\prime}-\sum_{i=0}^{1} y^{\left(1 / 2 \cdot i_{i j}\right)}(0+) \delta^{\left(-1 / 2 \cdot i_{i}\right)}(t)$

leading to a free term with LT given by

$F_{f}(s)=\frac{y(0+)+y^{(1 / 2)}(0+) s^{-1 / 2}}{s+a}$.

Obviously different from the usual that we obtain by putting $y^{(1 / 2)}(0+)=0$.

\section{Conclusions}

We approached the initial conditions problem from a sequential point of view and working in the space of the functions verifying the Watson-Doetsch lemma. The solution we obtained showed that, in general, we must speak in initial functions instead of initial values, in the sense that the initial values originates the presence of initial functions that influence the solution, not only at $t=0$, but for all $t \geqslant 0$. With this point of view, we obtained a broad set of initial conditions that we can choose according to our interests or facility in solving a specific problem. The context of the Watson-Doetsch lemma cover most of the functions we are interested in applications.

\section{Appendix A. On the differintegration}

\section{A.1. One-step differintegration}

We begin by considering the formulations of the differintegration based on the general double convolution:

$D^{\alpha} x(t)=x(t) * \delta^{(n)}(t) * \delta^{(-v)}(t)$, where $D$ means derivative $(\alpha>0)$ or integral $(\alpha<0), n \in Z, 0 \leqslant v<1, \alpha=n-v$,

$\delta_{ \pm}^{(n)}(t)= \begin{cases}D^{(n)} \delta(t), & n \geqslant 0 \\ \pm \frac{t^{(-n-1)}}{(-n-1) !} u( \pm t), & n<0\end{cases}$

with $\delta(t)$ as the impulse Dirac distribution, and

$\delta_{ \pm}^{(-v)}(t)= \begin{cases} \pm \frac{t^{v-1}}{\Gamma(v)} u( \pm t), & 0<v<1, \\ \delta(t), & v=0,\end{cases}$

where + stand for forward and - for backward differintegrations. As it is clear, we have three possibilities in the computation of the differintegration according to the way how we use the associative property of the convolution. The following association:

$x^{(\alpha)}(t)=x(t) *\left\{\delta^{(n)}(t) * \delta^{(-v)}(t)\right\}$

the Generalised Functions differintegration-also called Cauchy differintegration, mainly in complex variable formulation [10]. It is not hard to see that, if we retain the finite part, we can write

$$
\begin{aligned}
\delta^{(\alpha)}(t) & =\left\{\delta^{(n)}(t) * \delta^{(-v)}(t)\right\} \\
& = \begin{cases}\frac{t^{-\alpha-1}}{\Gamma(-\alpha)} u(t), & v \neq 0, \\
\delta^{(n)}(t), & v=0 .\end{cases}
\end{aligned}
$$

For reasons that will be clear in section in the following, we will consider the derivative case.

Alternatively to convolutional definition of derivative, we can use the Grünwald-Letnikov, that is a generalisation of the integer order derivative definition. Let $x(t)$ a limited function and $\alpha>0$. We define derivative of order $\alpha$ by

$x_{+}^{(\alpha)}(t)=\lim _{h \rightarrow 0^{+}} \frac{\sum_{k=0}^{\infty}(-1)^{k}\left(\begin{array}{l}\alpha \\ k\end{array}\right) x(t-k h)}{h^{\alpha}}$.

For well behaved functions these definitions may also be valid for $\alpha<0$ (integration). For right-hand signals the summation is carried only over a finite number of 
terms. In particular, for causal signals, we have

$x_{+}^{(\alpha)}(t)=\lim _{h \rightarrow 0^{+}} \frac{\sum_{k=0}^{N}(-1)^{k}\left(\begin{array}{l}\alpha \\ k\end{array}\right) x(t-k h)}{h^{\alpha}}$

with $N$ equal to the integer part of $t / h$. For the left-hand signals, we have

$x_{-}^{(\alpha)}(t)=\lim _{h \rightarrow 0^{+}} \mathrm{e}^{\mathrm{j} \pi \alpha} \frac{\sum_{k=0}^{\infty}(-1)^{k}\left(\begin{array}{l}\alpha \\ k\end{array}\right) x(t+k h)}{h^{\alpha}}$

and if the signal is anti-causal

$x_{-}^{(\alpha)}(t)=\lim _{h \rightarrow 0^{+}} \mathrm{e}^{\mathrm{j} \pi \alpha} \frac{\sum_{k=0}^{N}(-1)^{k}\left(\begin{array}{l}\alpha \\ k\end{array}\right) x(t+k h)}{h^{\alpha}}$.

\section{A.2. Multi-step differintegration}

Returning to the convolution in (1) we may consider other associations. With

$x^{(\alpha)}(t)=\delta^{(n)}(t) *\left\{x(t) * \delta^{(-v)}(t)\right\}$

we obtain the Riemann-Liouville differintegration. As seen, we proceed sequentially by the computation of a $v$ order integration, followed by $n$ derivative computations. With the association:

$x^{(\alpha)}(t)=\left\{x(t) * \delta^{(n)}(t)\right\} * \delta^{(-v)}(t)$

we obtain the Caputo differintegration. Here and relatively to the previous case, we inverted the process, beginning by $n$ derivative computations followed by a $v$ order integration. Of course, other possibilities do exist as it is the Miller-Ross sequential differintegration [9]:

$x^{(\alpha)}(t)=D^{\alpha} x(t)=\left[\prod_{i=1}^{N} D^{\sigma}\right] x(t)$

with $\alpha=N \sigma$. This is a special case of multi-step case proposed by Samko et al. [14] and based on the Riemann-Liouville definition:

$x^{(\alpha)}(t)=\left[\prod_{i=1}^{N} D^{\sigma_{i}}\right] x(t)$

with

$\alpha=\left[\sum_{i=1}^{N} \sigma_{i}\right]-1 \quad$ and $\quad 0<\sigma_{i} \leqslant 1$.
It is a simple task to obtain other decompositions of $\sigma$, leading to valid definitions. For the GrünwaldLetnikov definitions we can obtain similar definitions. We define the $v$-order derivative by (A.6) with $\alpha=v$. We can write, for example:

$x^{(\alpha)}(t)=D^{n} x^{(v)}(t)$

or, similarly

$x^{(\alpha)}(t)=D^{v} x^{(n)}(t)$.

These definitions suggest us that, to compute a $\alpha$ derivative, we have infinite ways, depending on the steps that we follow to go from 0 (or $-v$ ) to $\alpha$; that is we express $\alpha$ as a summation of $N$ reals $\sigma_{i}(i=0, \ldots, N-1)$, with the $\sigma_{i}$ not necessarily less or equal to one.

\section{References}

[1] M. Davison, C. Essex, Fractional differential equations and initial value problems, Math. Sci. 23 (1998) 108-116.

[2] J.C. Ferreira, Introduction to the Theory of Distributions, Pitman Monographs and Surveys in Pure and Applied Mathematics, July 1997.

[3] P. Henrici, Applied and Computational Complex Analysis, Vol. 2, Wiley, New York, 1991, pp. 389-391.

[4] M. Itagaki, A. Taya, K. Watanabe, K. Noda, Deviations of capacitive and inductive loops in the electrochemical impedance of a dissolving iron electrode, Anal. Sci. 18 (June 2002) 641-644.

[5] C.F. Lorenzo, T.T. Hartley, Initialisation conceptualization, and application in the generalized fractional calculus, NASA TP-1998-208415, 1998.

[6] C.F. Lorenzo, T.T. Hartley, Initialised fractional calculus, Internat. J. Appl. Math. 3 (3) (2000) 249-265.

[7] C.F. Lorenzo, T.T. Hartley, Initialization in fractional order systems, Proceedings of the European Control Conference, Porto, Portugal, 2001, pp. 1471-1476.

[8] P.J. Mahon, G.L. Paul, S.M. Kesshishian, A.M. Vassallo, Measurement and modelling of high-power performance of carbon-based supercapacitors, J. Power Sources 91 (2000) 68-76.

[9] K.S. Miller, B. Ross, An Introduction to the Fractional Calculus and Fractional Differential Equations, Wiley, Inc., New York, 1993.

[10] K. Nishimoto, Fractional Calculus, Descartes Press Co., Koriyama, 1989.

[11] M.D. Ortigueira, Introduction to fractional signal processing. Part 1: continuous-time systems, IEE Proceedings on Vision, 
Image and Signal Processing, Vol. 1, February 2000, pp. $62-70$.

[12] M.D. Ortigueira, Introduction to fractional linear systems: interconversions, Proceedings of the Fifth Ibero-American Symposium on Pattern Recognition, Lisbon, September 11-13, 2000, pp. 729-739.

[13] I. Podlubny, Fractional Differential Equations, Academic Press, San Diego, 1999.
[14] S.G. Samko, A.A. Kilbas, O.I. Marichev, Fractional Integrals and Derivatives-Theory and Applications, Gordon and Breach, London, New York, 1987.

[15] S. Westerlund, Dead Matter has Memory, Causal Consulting, Kalmar, Sweden, 2002.

[16] A.H. Zemanian, Distribution Theory and Transform Analysis, Dover, New York, 1987. 